\title{
Imaging of Patients with Suspected Large-Vessel Occlusion at Primary Stroke Centers: Available Modalities and a Suggested Approach
}

\author{
(D) M.A. Almekhlafi, (D)W.G. Kunz, DB.K. Menon, DR.A. McTaggart, (D)M.V. Jayaraman, (DB.W. Baxter, (DDD. Heck, (DDD. Frei, \\ (D)C.P. Derdeyn, (D)T. Takagi, (D)A.H. Aamodt, DI.M.R. Fragata, DM.D. Hill, DA.M. Demchuk, and (D) M. Goyal
}

\begin{abstract}
SUMMARY: The overwhelming benefit of endovascular therapy in patients with large-vessel occlusions suggests that more patients will be screened than treated. Some of those patients will be evaluated first at primary stroke centers; this type of evaluation calls for standardizing the imaging approach to minimize delays in assessing, transferring, and treating these patients. Here, we propose that CT angiography (performed at the same time as head CT) should be the minimum imaging approach for all patients with stroke with suspected large-vessel occlusion presenting to primary stroke centers. We discuss some of the implications of this approach and how to facilitate them.
\end{abstract}

ABBREVIATIONS: CSC = comprehensive stroke center; DIDO = door-in-door-out time interval; EVT = endovascular thrombectomy; LAMS = Los Angeles Motor Scale; LVO = large-vessel occlusion; PSC = primary stroke center; $\mathrm{P}_{\mathrm{PSC}} 2 \mathrm{P}_{\mathrm{CSC}}=$ picture at the primary stroke center to puncture at the comprehensive stroke center time

schemic stroke due to large-vessel occlusion (LVO) substantially contributes to morbidity and mortality. Despite the improvements in systems of care and the development of effective interventions, a minority of eligible patients receive acute therapies. ${ }^{1}$ To increase patients' access to treatment, stroke centers are certified as primary (ie, capable of administering intravenous thrombolytics) and comprehensive (ie, capable of administering thrombolytics and endovascular thrombectomy [EVT]).

The evidence for the benefit from EVT in patients with LVO is overwhelming, with a number needed to treat of 2.6 to reduce functional disability on the modified Rankin Scale by at least 1 level. ${ }^{2}$ This benefit is evident across all subgroups and is indepen-

Received October 5, 2018; accepted after revision December 6.

From the Departments of Radiology and Clinical Neurosciences (M.A.A., B.K.M., M.D.H., A.M.D., M.G.), University of Calgary, Calgary, Alberta, Canada; Department of Radiology (W.G.K.), University Hospital, Ludwig Maximilian University of Munich, Munich, Germany; Departments of Neurology, Diagnostic Imaging, and Neurosurgery (R.A.M., M.V.J.), Warren Alpert Medical School of Brown University, Providence, Rhode Island; Department of Radiology (B.W.B.), Erlanger Medical Center, Chattanooga, Tennessee; Department of Radiology (D.H.), Forsyth Medical Center Winston Salem, North Carolina; Swedish Medical Center (D.F.), Denver, Colorado; Department of Radiology (C.P.D.), University of lowa Hospitals and Clinics, lowa City, lowa; Department of Neurosurgery (T.T.), Hyogo College of Medicine, Nishinomiya, Hyögo, Japan; Department of Neurology (A.H.A.), Oslo University Hospital, Oslo, Norway; and Department of Neuroradiology (I.M.R.F.), Centro Hospitalar Lisboa Central, Lisbon, Portugal.

Please address correspondence to Mayank Goyal, MD, FRCPC, Department of Radiology, Seaman Family MR Research Centre, Foothills Medical Centre, 1403 29th St. NW, Calgary AB T2N2T9, Canada; e-mail: mgoyal@ucalgary.ca; @AlmekhlafiMa; @WolfganagGKunzMD; @bijoymenon; @mobilestroke4U; @mayank_G0; @mihill68; @DCNSNeuro

http://dx.doi.org/10.3174/ajnr.A5971 dent of age, stroke severity, or the extent of early ischemic changes on imaging. This effect makes most patients with LVO potentially eligible for EVT within 6 hours from onset. While early treatment is associated with greater benefit, ${ }^{3}$ EVT is still associated with better outcomes in selected patients in the 6- to 24-hour time window. ${ }^{4,5}$ Therefore, it is critical that all potential EVT candidates be screened for LVO rapidly and then rushed to comprehensive stroke centers (CSCs) for EVT.

Because many patients will first arrive at primary stroke centers (PSCs), the improvement of workflow processes at PSCs to identify EVT candidates is a priority. To achieve this, we recommend that all patients triaged as having potential LVO (eg, the Los Angeles Motor Scale [LAMS] score ${ }^{6}$ is $\geq 4$ ) receive a standardized, 1-stop imaging with noncontrast head CT and CTA at the same time to confirm the presence of LVO and initiate the transfer process. Key challenges to this approach include reliable head CT interpretation and detection of LVO on CTA by nonexpert readers. Advanced imaging, such as multiphase CTA or CTP, can help overcome challenges related to variability in head CT interpretation. Multiphase CTA simplifies LVO detection even for nonexpert readers ${ }^{7}$ and provides collateral assessment and core/penumbra estimation. ${ }^{8,9}$ While evidence from the Efficacy and Safety of MRI-Based Thrombolysis in Wake-Up Stroke trial supports MR imaging-based selection of patients with unknown time of onset, ${ }^{10}$ CT-based paradigms are more widely available and faster and have fewer contraindications compared with MR imaging, making them more appropriate for the PSC setting. ${ }^{11}$

Herein, we discuss the driving principles behind our proposed 
imaging approach at PSCs and how they relate to the core concepts of stroke therapy. These recommendations are not meant to be binding, but they aim to engage physicians from all relevant disciplines in both PSCs and CSCs and policymakers in a discussion to streamline the imaging approach in patients with suspected LVO in PSCs.

\section{Appropriateness of Transfer}

A critical PSC-related challenge is the appropriateness of transferring patients with suspected LVO. We have recently debated this topic and proposed a classification scheme to assess the appropriateness of patient transfer for EVT. ${ }^{12}$ Some of the clinical and imaging characteristics of patients who are not candidates for transfer include those with the following:

1) Completed infarct in which recanalization will be both futile and risky ${ }^{13}$

1) High likelihood of reperfusion with intravenous thrombolytic therapy (distal occlusion, small thrombus, favorable thrombus characteristics) $)^{14,15}$

3) Severe comorbidities or poor premorbid status.

While PSCs should err toward overtransferring rather than undertransferring because of the significant effect size of EVT, the above-mentioned characteristics highlight the role of advanced imaging in modern stroke care.

\section{Time Matters}

Fast treatment is essential for good stroke outcome. This is emphasized in the pooled analyses in the highly effective reperfusion using multiple endovascular devices (HERMES) collaboration. ${ }^{3}$ Hence, one must balance the time spent on obtaining and interpreting imaging and the value and relevance of the information it provides. At the CSC level, use of CTA has been linked to shorter onset-to-treatment times. ${ }^{16}$ The need for and feasibility of routinely performing advanced imaging at PSCs are less clear. While it will exhaust the limited resources of PSCs if CTA is performed on all patients with stroke without discrimination, there are merits to performing CTA in all patients with suspected LVO (based on the LAMS, NIHSS, presence of cortical signs, and so forth) at the same time as head CT. Patients not suspected of having LVO such as those with minor stroke symptoms or those who are not candidates for EVT, for example due to pre-existing disability or functional impairment, could be assessed with head CT alone as an initial step.

One approach to advanced imaging at the PSC level is to protocol the technique on the basis of the time from symptom onset. Patients who present early ( $<6$ hours) from onset undergo head CT/CTA, while those arriving at later times (6-24 hours from onset) or those with suspected LVO stroke on awakening also undergo CTP. Ultimately, a standardized, protocol-driven advanced imaging pathway is likely to be the most efficient strategy in triaging patients at the PSC.

\section{Technique and Technical Support Matter}

For advanced imaging to be used at PSCs, imaging protocols need to be well-developed, and qualified technologists need to be available to perform them around the clock. Results should be conveyed promptly to the managing physicians for the transfer pro- cess to be initiated rapidly. Also, images need to be accessible to the CSC team. These prerequisites may pose challenges for the PSC, given their limited resources, especially outside of the working hours. Teleradiology consult with CSCs may be a potential solution by using image transfer or a sharing platform or the cloud. Alternatively, automated postprocessing software can be used to immediately alert physicians to the presence of potential thrombectomy candidates. Such software needs to be fast and adequately tested, and personnel involved need to be trained to recognize factors that may result in erroneous interpretation (eg, patient motion). Another pitfall common to images obtained at the PSC is the risk of decay (progression of infarction during transfer), requiring repeat imaging based on a variety of clinical characteristics (Figure). ${ }^{17}$

\section{Door-In-Door-Out Is Critical}

Workflow time metrics influence the outcomes of patients with LVO stroke who initially present to PSCs (Figure). These include the time from arrival at the PSC emergency door to the start of intravenous thrombolytic therapy (door-to-needle time), the time from arriving at the PSC door until the patient leaves the PSC for a CSC (door-in-door-out time interval [DIDO]), and the time from imaging at the PSC to the arterial puncture time at the CSC $\left(\mathrm{P}_{\mathrm{PSC}} 2 \mathrm{P}_{\mathrm{CSC}}\right) \cdot{ }^{18}$ Some of these metrics depend on the efficiency of workflow in the PSC (door-to-needle time, DIDO), while the $\mathrm{P}_{\mathrm{PSC}} 2 \mathrm{P}_{\mathrm{CSC}}$ integrates the time needed for transfer and that of any additional imaging performed at the CSC. The DIDO metric has become the chief measure for efficient management of patients with LVO stroke within PSCs. Shortening the DIDO is feasible and results in a shorter time to groin puncture. ${ }^{19} \mathrm{~A}$ target DIDO of $\leq 45$ minutes was achieved by McTaggart et al. ${ }^{19}$ Health care systems should aim to achieve a DIDO as short as safely possible in their stroke network based on the available resources.

The same paramedic team that brings the patient to the PSC should accompany the patient during imaging and transfer to the CSC if needed, to achieve an ultrashort DIDO. This arrangement has many advantages, including the continuity of care, because the same personnel at the first medical contact will be more likely to pick up any improvement or deterioration. This approach will save time and reflect positively on patient outcomes. One disadvantage is making certain that ambulance team is unavailable for other emergencies during that entire time. However, if an ultrashort DIDO can be implemented and consistently achieved, the door-to-decision time at the PSC will be extremely short and it will be an incentive for the paramedics to remain at the patient's side. To facilitate this ultrashort DIDO, the decision to transfer to a CSC will need to be made directly after CTA once the presence of proximal occlusion is confirmed. The complete interpretation of NCCT and CTA with formal written reports can follow later and should not be required for transfer. Built-in software for automated ASPECTS interpretation, LVO detection, and perfusion imaging (for patients with a late window) will expedite this process.

In summary, to maximize the workflow efficiency and decision-making at the PSC level, the same paramedic crew should accompany the patient from first medical contact, during the stay in PSC, and to the CSC if needed. Imaging modalities at the PSC should do the following:

AJNR Am J Neuroradiol 40:396-400 Mar 2019 www.ajnr.org 

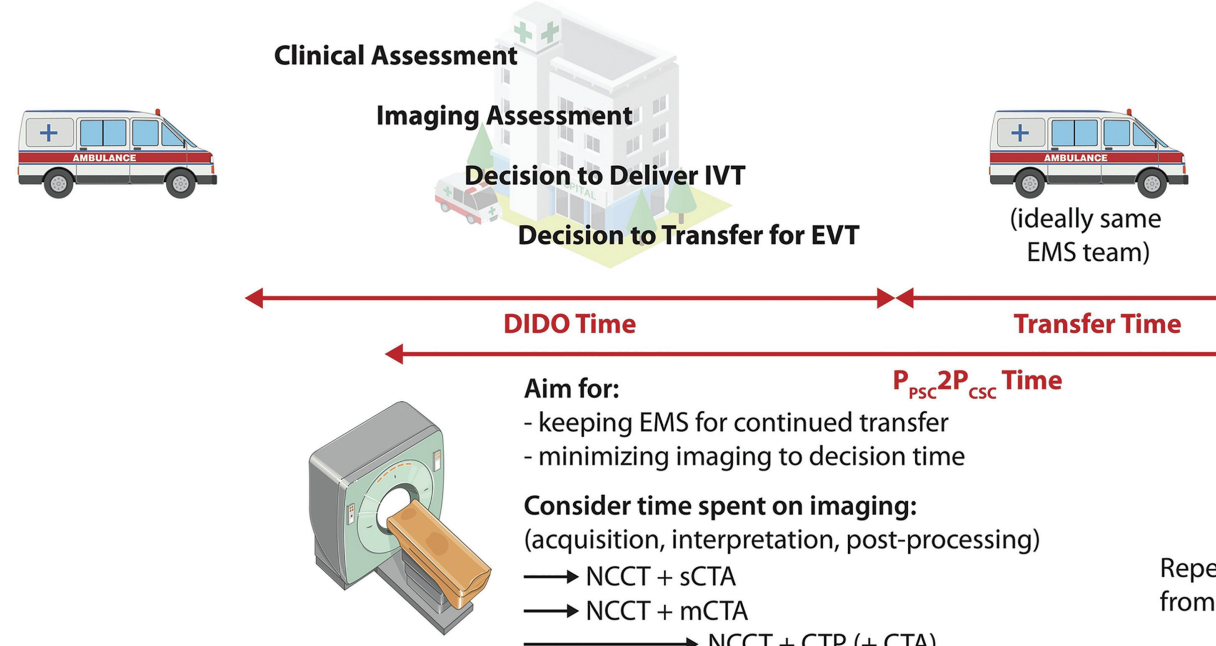

DIDO Time

$\mathbf{P}_{\mathrm{PsC}} 2 \mathrm{P}_{\mathrm{csc}}$ Time

- keeping EMS for continued transfer

- minimizing imaging to decision time

Consider time spent on imaging:

(acquisition, interpretation, post-processing)

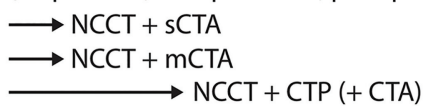

Repeat imaging depending upon time from first imaging to CSC arrival*

FIGURE. The workflow of patients with LVO stroke arriving first at a primary stroke center. Once patients arrive at the PSC, steps that can introduce delay in the door-in-door-out time interval include clinical and imaging assessments and the time spent on each of the various imaging modalities (the length of the arrows corresponds to the relative duration needed to complete, process, and interpret each technique). Following imaging, the decisions to administer intravenous thrombolytics (IVT) and whether to transfer to comprehensive stroke centers for endovascular thrombectomy can introduce some delay. Transfer to CSCs is preferably done by the same Emergency Medical Service (EMS) team that brought the patient to the PSC. Once the patient arrives at a CSC, repeat imaging can be considered in selected patients $\left({ }^{*}\right)$, given the low likelihood of recanalization of LVO with intravenous thrombolytics and the variability of CT ASPECTS decay. Repeat imaging at the CSC will further delay the time interval from imaging at the PSC to arterial puncture. mCTA indicates multiphase CTA; sCTA, single-phase CTA; angio, angiography.

Imaging options, advantages, and limitations at primary stroke centers

\begin{tabular}{|c|c|c|}
\hline Imaging Modality & Pros & Cons \\
\hline Plain CT only & Highly time-efficient & $\begin{array}{l}\text { Detection of LVO is not reliable. } \\
\text { Dense MCA sign is not reliable } 20 \\
\text { Variability in ASPECTS reading, especially in the very early } \\
\text { time window }{ }^{21}\end{array}$ \\
\hline $\begin{array}{l}\text { CT plus single-phase } \\
\text { CTA (arch to vertex) }\end{array}$ & $\begin{array}{l}\text { Exact occlusion site } \\
\text { Basic assessment of collaterals } \\
\text { Roadmap for EVT procedure }\end{array}$ & $\begin{array}{l}\text { Considerable risk of collateral underestimation if } \\
\text { acquisition timed too early } \\
\text { Distal occlusions might be missed; challenges with training } \\
\text { and maintaining competence for technologists in } \\
\text { low-volume community settings }\end{array}$ \\
\hline CT plus multiphase CTA & $\begin{array}{l}\text { Robust collateral assessment (independent } \\
\text { of timing) } \\
\text { Diagnosis of LVO in inexperienced readers } \\
\text { Easier detection of distal occlusions }{ }^{7} \\
\text { No additional contrast needed } \\
\text { Can be done on any modern scanner }\end{array}$ & Collateral assessment may require training \\
\hline CT plus CTP & $\begin{array}{l}\text { Easier identification of patients eligible for EVT } \\
\text { Easier identification of stroke mimics in the } \\
\text { absence of } \mathrm{LVO}^{22}\end{array}$ & $\begin{array}{l}\text { More sensitive to patient motion } \\
\text { Time-consuming; may create a bottleneck in a workflow } \\
\text { wherein the paramedic team that brings the patient in } \\
\text { is the same team that takes the patient to the CSC } \\
\text { Limited comparability of CTP results across protocols and } \\
\text { postprocessing software } 23,24 \\
\text { Higher contrast and radiation dose needed } \\
\text { CTP pitfalls }{ }^{25} \\
\text { Cost of additional postprocessing software, optional }\end{array}$ \\
\hline
\end{tabular}

1) Rule out intracerebral hemorrhage

2) Identify large-vessel occlusion

3) Identify a large-infarction core (eg, ASPECTS $<6$ or core $>50 \mathrm{~mL}$; consider eloquence of the affected or spared brain regions) and the infarction dynamics/decay (collaterals versus the effect of the $\left.\mathrm{P}_{\mathrm{PSC}} 2 \mathrm{P}_{\mathrm{CSC}}\right)$.
The Table compares the various imaging options at the PSC level.

\section{DISCUSSION}

The choice of imaging technique at the PSC implies a certain threshold for CSC transfer based on the information that each technique provides. A minimalist imaging approach may set 
lower thresholds for transfer that consider all suspected patients but come at the expense of relatively high inappropriate transfers. The use of perfusion imaging implies strict thresholds based on the presence of a target mismatch to allow the transfer. This perfusion mismatch paradigm comes at the expense of excluding patients with proved benefit from EVT as shown by the Multicenter Randomized Clinical Trial of Endovascular Treatment for Acute Ischemic Stroke in the Netherlands ${ }^{26}$ and the Trial and Cost Effectiveness Evaluation of Intra-arterial Thrombectomy in Acute Ischemic Stroke, ${ }^{27}$ which selected patients on the basis of NCCT and the presence of proximal occlusion. Thus, the 2018 American Stroke Association guidelines did not recommend the use of perfusion imaging for selecting patients for EVT in the $<6$-hour time window. ${ }^{28}$ Advanced imaging also comes at the expense of DIDO.

The imaging approaches in a given institution are dependent on its available resources, expected patient volume, the distance to the CSC (the closer the CSC, the less the need to perform advanced imaging at the PSC), and the availability of an imagingsharing platform with the CSC. The imaging approach can be revisited, depending on the door-to-needle time and DIDO, and according to infrastructure and resources. In addition, the approach needs to be tailored to the patient's onset time.

We support the implementation of vascular imaging at the PSC level as a goal that all stroke systems of care should achieve. While it is estimated that around $10 \%-20 \%$ of LVOs will recanalize with intravenous thrombolytics en route to the $\mathrm{CSC},{ }^{29}$ the rate of ASPECTS/collateral decay is not known. One study described ASPECTS decay in $19.6 \%$ of patients transferred to CSCs for EVT. ${ }^{30,31}$ This was noted in patients with poor baseline ASPECTS and poor collateral flow and higher baseline stroke severity, irrespective of the onset-to-imaging time or the duration of the transfer.

While we propose that CT and CTA need to be the basis of imaging in all patients with suspected LVO, the limitations of these imaging modalities need to be considered. In addition to the points discussed earlier (Table), the reliability of CT ASPECTS in the early time window ( $<3$ hours) may lead to excluding/including inappropriate transfers for EVT. ${ }^{21}$ A training Web site for ASPECTS is available to tackle many of the perceived challenges related to CT (and CTA) interpretation in the acute stroke setting (www.aspectsinstroke.com). Single-phase CTA assessment of collaterals could be clouded by poor injection timing. Here, multiphase CTA has a clear advantage. While the Evaluation Study of Congestive Heart Failure and Pulmonary Artery Catheterization Effectiveness trial enrolled patients up to 12 hours on the basis of CTA (or multiphase CTA) collaterals, ${ }^{32}$ patient selection based on collateral imaging has not been directly compared with perfusion imaging for selecting patients in the 6- to 24-hour time window. Prospective cohorts have shown that multiphase CTA fared better than CTP in selecting patients within 6 hours from onset. ${ }^{8}$

\section{Future Directions and Conclusions}

The advent of EVT in patients with LVO has opened the field for innovation and technology. One promising aspect is the use of automated aids for decision-making. ${ }^{33}$ Some of the developed software tools were found to be noninferior to interpretation of the ASPECTS by a neuroradiologist. ${ }^{34}$ There is innovation in the prehospital detection of LVO. The volumetric impedance phase shift spectroscopy (VIPS) device (Cerebrotech Medical Systems, Pleasanton, California) has shown $>90 \%$ sensitivity and specificity for detecting severe strokes. ${ }^{35}$ If this technology or similar ones reliably identify patients with LVO in the field, it will provide the opportunity for rational, direct transfer of some patients to nearby CSCs, eliminating the need for stopping at PSCs.

In conclusion, EVT has proved efficacy for a wide range of patients with LVO stroke and in selected cases up to 24 hours from onset. While PSCs have increased the proportion of patients with stroke receiving thrombolytic therapy, delays can be encountered until patients with LVO are identified and transferred from the PSC to the CSC. Therefore, any extra steps need to be carefully weighed. The use of CTA (especially multiphase) at the PSC level has many advantages in expediting the transfer of appropriate patients to CSCs. However, the routine implementation of CTA requires resources and training in addition to the infrastructure for sharing images with CSCs.

Disclosures: Blaise W. Baxter-UNRELATED: Consultancy: Penumbra, Medtronic, Stryker, Route 92, Viz.ai, 880 Medical, Comments: stroke-treatment consulting; Payment for Lectures Including Service on Speakers Bureaus: Penumbra, Comments: stroke-treatment talks; Patents (Planned, Pending or Issued): patent No. 9526863, Comments: devices and methods for perfusion therapy; Stock/Stock Options: Penumbra, Viz.ai. Colin P. Derdeyn—UNRELATED: Consultancy: Penumbra, NoNO Inc., genae, Comments: Data and Safety Monitoring Board for the MIND trial, Data and Safety Monitoring Board for the ESCAPE NA and FRONTIER trials, Data and Safety Monitoring Board for the TIGERTRIEVER trial*; Grants/Grants Pending: Siemens*; Stock/Stock Options: Pulse Therapeutics, Comments: stroke-treatment device. Michael D. Hill-UNRELATED: Consultancy: Boehringer Ingelheim, Bristol-Myers Squibb, Pfizer, NoNO Inc., Comments: consultant and continuing medical education teaching, some paid and some not*; Grants/Grants Pending: NoNO Inc., Bayer Canada, Boehringer Ingelheim, Medtronic, Stryker, Comments: grants to the University of Calgary for ongoing clinical trials*; Stock/Stock Options: Calgary Scientific, Comments: stock ownership.* Mayank Goyal—RELATED: Other: GE Healthcare, Comments: licensing agreement for systems of stroke diagnosis; UNRELATED: Consultancy: Medtronic, Stryker, MicroVention, Mentice, Comments: education and advice related to acute stroke; Grants/Grants Pending: Stryker, Comments: unrestricted research grant to the University of Calgary for the UNMASK EVT study.* Ryan A. McTaggart-UNRELATED: Consultancy: Stryker. Mahesh V. JayaramanRELATED: Consulting Fee or Honorarium: Medtronic, Comments: 1-time fee for speaking at the International Stroke conference 2018 on stroke systems of care. Donald Heck—UNRELATED: Consultancy: Stryker; Payment for Lectures Including Service on Speakers Bureaus: Stryker; Payment for Development of Educational Presentations: Stryker. Donald Frei-UNRELATED: Consultancy: Penumbra, Stryker, Genentech; Payment for Lectures Including Service on Speakers Bureaus: Stryker, Genentech, Penumbra; Payment for Development of Educational Presentations: Genentech, National Stroke Association; Stock/Stock Options: Penumbra. Andrew M. Demchuk—RELATED: Consulting Fee or Honorarium: Medtronic, Comments: for continuing medical education events. *Money paid to the institution.

\section{REFERENCES}

1. Messé SR, Khatri P, Reeves MJ, et al. Why are acute ischemic stroke patients not receiving IV tPA? Results from a national registry. Neurology 2016;87:1565-74 CrossRef Medline

2. Goyal M, Menon BK, van Zwam WH, et al; HERMES collaborators. Endovascular thrombectomy after large-vessel ischaemic stroke: a meta-analysis of individual patient data from five randomised trials. Lancet 2016;387:1723-31 CrossRef Medline

3. Saver JL, Goyal M, van der Lugt A, et al; HERMES collaborators. Time to treatment with endovascular thrombectomy and outcomes from ischemic stroke: a meta-analysis. JAMA 2016;316:1279-88 CrossRef Medline

4. Albers GW, Marks MP, Kemp S, et al; DEFUSE 3 Investigators. Thrombectomy for stroke at $\mathbf{6}$ to 16 hours with selection by perfusion imaging. N Engl J Med 2018;378:708-18 CrossRef Medline 
5. Nogueira RG, Jadhav AP, Haussen DC, et al; DAWN Trial Investigators. Thrombectomy 6 to 24 hours after stroke with a mismatch between deficit and infarct. $N$ Engl J Med 2018;378:11-21 CrossRef Medline

6. Llanes JN, Kidwell CS, Starkman S, et al. The Los Angeles Motor Scale (LAMS): a new measure to characterize stroke severity in the field. Prehosp Emerg Care 2004;8:46-50 CrossRef Medline

7. Amy Y, Zerna C, Assis Z, et al. Multiphase CT angiography increases detection of anterior circulation intracranial occlusion. Neurology 2016;87:609-16 CrossRef Medline

8. Menon BK, d'Esterre CD, Qazi EM, et al. Multiphase CT angiography: a new tool for the imaging triage of patients with acute ischemic stroke. Radiology 2015;275:510-20 CrossRef Medline

9. Tan BY, Wan-Yee K, Paliwal P, et al. Good intracranial collaterals trump poor ASPECTS (Alberta Stroke Program Early CT Score) for intravenous thrombolysis in anterior circulation acute ischemic stroke. Stroke 2016;47:2292-98 CrossRef Medline

10. Thomalla G, Simonsen CZ, Boutitie F, et al; WAKE-UP Investigators. MRI-guided thrombolysis for stroke with unknown time of onset. N Engl J Med 2018;379:611-22 CrossRef Medline

11. Jovin TG. MRI-guided intravenous alteplase for stroke: still stuck in time. $N$ Engl J Med 2018;379:682-83 CrossRef Medline

12. Goyal M, Menon BK, Wilson AT, et al. Primary to comprehensive stroke center transfers: appropriateness, not futility. Int J Stroke 2018;13:550-53 CrossRef Medline

13. Liebeskind DS, Bracard S, Guillemin F, et al. eTICI reperfusion: defining success in endovascular stroke therapy. J Neurointerv Surg 2018:neurintsurg-2018-014127

14. Mishra SM, Dykeman J, Sajobi TT, et al. Early reperfusion rates with IV tPA are determined by CTA clot characteristics. AJNR Am J Neuroradiol 2014;35:2265-72 CrossRef Medline

15. Menon BK, Al-Ajlan FS, Najm M, et al; INTERRSeCT Study Investigators. Association of clinical, imaging, and thrombus characteristics with recanalization of visible intracranial occlusion in patients with acute ischemic stroke. JAMA 2018;320:1017-26 CrossRef Medline

16. Menon BK, Campbell BC, Levi C, et al. Role of imaging in current acute ischemic stroke workflow for endovascular therapy. Stroke 2015;46:1453-61 CrossRef Medline

17. Sun $\mathrm{CH}$, Connelly K, Nogueira RG, et al. ASPECTS decay during inter-facility transfer predicts patient outcomes in endovascular reperfusion for ischemic stroke: a unique assessment of dynamic physiologic change over time. J Neurointerv Surg 2015;7:22-26 CrossRef Medline

18. Sun CH, Nogueira RG, Glenn BA, et al. "Picture to puncture": a novel time metric to enhance outcomes in patients transferred for endovascular reperfusion in acute ischemic stroke. Circulation 2013;127:1139-48 CrossRef Medline

19. McTaggart RA, Yaghi S, Cutting SM, et al. Association of a primary stroke center protocol for suspected stroke by large-vessel occlusion with efficiency of care and patient outcomes. JAMA Neurol 2017;74:793-800 CrossRef Medline

20. Kharitonova T, Thorén M, Ahmed N, et al ; SITS investigators. Disappearing hyperdense middle cerebral artery sign in ischaemic stroke patients treated with intravenous thrombolysis: clinical course and prognostic significance. J Neurol Neurosurg Psychiatry 2009;80:273-78 CrossRef Medline

21. Bal S, Bhatia R, Menon BK, et al. Time dependence of reliability of noncontrast computed tomography in comparison to computed tomography angiography source image in acute ischemic stroke. Int J Stroke 2015;10:55-60 CrossRef Medline

22. Hopyan J, Ciarallo A, Dowlatshahi D, et al. Certainty of stroke diagnosis: incremental benefit with CT perfusion over noncontrast CT and CT angiography. Radiology 2010;255:142-53 CrossRef Medline

23. Austein F, Riedel C, Kerby T, et al. Comparison of perfusion CT software to predict the final infarct volume after thrombectomy. Stroke 2016;47:2311-17 CrossRef Medline

24. Bivard A, Kleinig T, Miteff F, et al. Ischemic core thresholds change with time to reperfusion: a case control study. Ann Neurol 2017;82: 995-1003 CrossRef Medline

25. Goyal M, Menon BK, Derdeyn CP. Perfusion imaging in acute ischemic stroke: let us improve the science before changing clinical practice. Radiology 2013;266:16-21 CrossRef Medline

26. Berkhemer OA, Fransen PS, Beumer D, et al. A randomized trial of intraarterial treatment for acute ischemic stroke. NEngl J Med 2015; 372:11-20 CrossRef Medline

27. Bracard S, Ducrocq X, Mas JL, et al; THRACE investigators, Mechanical thrombectomy after intravenous alteplase versus alteplase alone after stroke (THRACE): a randomised controlled trial. Lancet Neurol 2016;15:1138-47 CrossRef Medline

28. Powers WJ, Rabinstein AA, Ackerson T, et al; American Heart Association Stroke Council. 2018 Guidelines for the Early Management of Patients with Acute Ischemic Stroke: A Guideline for Healthcare Professionals from the American Heart Association/American Stroke Association. Stroke 2018;49:e46-110 CrossRef Medline

29. Tsivgoulis G, Katsanos AH, Schellinger PD, et al. Successful reperfusion with intravenous thrombolysis preceding mechanical thrombectomy in large-vessel occlusions. Stroke 2018;49:232-35 CrossRef Medline

30. Boulouis G, Lauer A, Siddiqui AK, et al. Clinical imaging factors associated with infarct progression in patients with ischemic stroke during transfer for mechanical thrombectomy. JAMA Neurol 2017; 74:1361-67 CrossRef Medline

31. Mokin M, Gupta R, Guerrero WR, et al. ASPECTS decay during inter-facility transfer in patients with large vessel occlusion strokes. J Neurointerv Surg 2017;9:442-44 CrossRef Medline

32. Goyal M, Demchuk AM, Menon BK, et al; ESCAPE Trial Investigators. Randomized assessment of rapid endovascular treatment of ischemic stroke. $N$ Engl J Med 2015;372:1019-30 CrossRef Medline

33. Lee EJ, Kim YH, Kim N, et al. Deep into the brain: artificial intelligence in stroke imaging. J Stroke 2017;19:277 CrossRef Medline

34. Nagel S, Sinha D, Day D, et al. e-ASPECTS software is non-inferior to neuroradiologists in applying the ASPECT score to computed tomography scans of acute ischemic stroke patients. Int J Stroke 2017;12:615-22 CrossRef Medline

35. Kellner CP, Sauvageau E, Snyder KV, et al. The VITAL study and overall pooled analysis with the VIPS non-invasive stroke detection device. J Neurointerv Surg 2018;10:1079-84 CrossRef Medline 